Vor. 48 (1993) [411-426]

\title{
LOCAL INTEGRAL METRICS AND DANIELL-LOOMIS INTEGRALS
}

\author{
M. Diaz Carrillo and H. Günzler
}

Recently Guerrero and the first author (Diaz Carrillo) proved an anologue to Daniell's extension process which works for arbitrary nonnegative linear functionals, without any continuity conditions. With the aid of Schäfke's local integral metrics we generalise this extension process and prove convergence theorems using a suitable local mean convergence, which can be traced back to Loomis.

\section{INTRODUCTION}

Recently in [3] an analogue to Daniell's extension process was given which works for arbitrary nonnegative linear functionals, without any continuity conditions. With the aid of Schäfke's local integral metrics [19] we generalise here this extension process and prove convergence theorems (which do not hold for $\bar{B}$ of [3]); this is possible using a suitable local mean convergence, which can be traced back to Loomis [13, p.179].

In Section 1 we recapitulate the Aumann-Schäfke integration theory for general integral metrics, prove an analogue of Lebesgue's convergence theorem, and introduce measurability. In Section 2, for general local integral metrics, more convergence theorems are obtained, extending results of [19] (we do not use $q((1 / n) h) \rightarrow 0$ or the restrictive 2 of [19]). In Section 3 a unified treatment of Riemann- $\mu$, abstract RiemannLoomis, Daniell and Bourbaki integrals is given.

With Schäfke $[16,17,18,19]$, most results extend to Banach space valued functions, using $x \cap t$ of [10], some even to function-valued integral metrics of [18, 19]. Part of our results of 3.E on $L(I \mid B)$ have been announced in [7].

\section{Notations}

We extend the usual + in $\overline{\mathbf{R}}:=\{-\infty\} \cup$ reals $\mathbb{R} \cup\{\infty\}$ to $\overline{\mathbf{R}} \times \overline{\mathbf{R}}$ by

$$
\begin{aligned}
& r+s:=0, \quad r+s:=\infty, \quad r+s:=-\infty, \text { if } r=-s \in\{\infty,-\infty\} \\
& r-s:=r+(-s) \text { et cetera. }
\end{aligned}
$$

Received 7 December 1992

Copyright Clearance Centre, Inc. Serial-fee code: 0004-9729/93 SA2.00+0.00. 
With $r \vee s:=\max (r, s), r \wedge s:=\min (r, s), r \cap t:=(r \wedge t) \vee(-t)$ if $0 \leqslant t \in \overline{\mathbb{R}}$ one still has for arbitrary $a, b, c, d \in \overline{\mathbb{R}}, 0 \leqslant t \in \overline{\mathbb{R}}$ the Birkhoff-inequalities

$$
\begin{aligned}
& |a \cap t-b \cap t| \leqslant 2(|a-b| \wedge t), \quad|a \wedge c-b \wedge c| \leqslant|a-b| \\
& |a \vee c-b \vee c| \leqslant|a-b|
\end{aligned}
$$

$$
|| a|-| b|| \leqslant|a-b| \leqslant|a-c|+|c-b|, \quad|(a+b)-(c+d)| \leqslant|a-c|+|b-d|
$$

(Aumann [3] $\left.\left({ }^{*} b,{ }^{*} c\right)\right) ;+,+,+$ are commutative, + is distributive with $0 \cdot( \pm \infty):=$ $0=:( \pm \infty) \cdot 0$, but not associative; $\dot{+}$ is associative. On the set $\overline{\mathbb{R}}$ of functions $f: X \rightarrow$ $\overline{\mathbb{R}}$ we define $=, \pm, \dot{+}, \wedge, \vee, \cap, \cdot,||, \leqslant$ pointwise on $X$ with $|f|(x):=|f(x)|, f \leqslant g$ if and only if $f(x) \leqslant g(x)$ for all $x \in X$. Define

$$
+M:=\{f \in M: 0 \leqslant f\} \quad \text { if } \quad M \subset \overline{\mathbb{R}}^{X} .
$$

$\left(k_{j}\right)=\left(k_{j}\right)_{j \in J}$ means a net with $k_{j} \in \overline{\mathbb{R}}^{X}, j \in J$ where $J$ is a set directed by $\leqslant$.

\section{Integral Metrics}

Standard assumptions in this and the following sections are

$$
X \text { is a non-empty set }, \quad 0 \in B \subset \overline{\mathbb{R}}^{X}, \quad q:+\overline{\mathbb{R}}^{X} \rightarrow+\overline{\mathbb{R}}:=[0, \infty] \text {. }
$$

For later reference and the benefit of the reader, we first collect some results mostly due to Aumann [1], Schäfke [16, 19]:

Definition 1: $q:+\overline{\mathbb{R}}^{X} \rightarrow+\overline{\mathbb{R}}$ is called an integral metric on $X$ if

$$
q(0)=0 \text { and } q(a) \leqslant q(b)+q(c) \text { if } a \leqslant b+c, \quad a, b, c \in+\overline{\mathbb{R}}^{X} \cdot([16])
$$

For an integral metric $q$ one gets with (3)

$$
|q(|a|)-q(|b|)| \leqslant q(|a-b|), \quad q(c) \leqslant q(d), \quad \text { if } a, b, c, d \in \overline{\mathbb{R}}^{X}, \quad 0 \leqslant c \leqslant d .
$$

$\left(\overline{\mathbb{R}}^{X}, q\right)$ is a topological space using $\left\{a \in \overline{\mathbb{R}}^{X}: q\left(\left|a-a_{0}\right|\right)<\varepsilon\right\}$ with - of (1).

Definition 2: $N_{q}:=\left\{k \in \overline{\mathbb{R}}^{X}: q(|k|)=0\right\}$ ( $q$-nulfunctions); if $M \subset \overline{\mathbb{R}}^{X}$ and $q$ is an integral metric on $X$, we define

$$
M^{q}:=\text { closure of } M \text { in }\left(\overline{\mathbb{R}}^{X}, q\right)
$$


( $q$-integrable functions, with respect to $M$ ).

LEMma 1. If $q$ is an integral metric on $X, 0 \in B \subset \overline{\mathbb{R}}^{X},{ }^{*} \in\{+,-, \ldots,||, s$. with $s \in \mathbb{R}$ \} and $B$ is closed with respect to ${ }^{*}$, for example $h+k \in B$ (respectively $h \cap|k|$, respectively $|h| \in B)$ if $h, k \in B$, then $B^{q}$ is also closed with respect to this *; $B \cup N_{q} \subset B^{q}=\left(B^{q}\right)^{q} . N_{q}$ is closed with respect to all operations from $\{\cdots\}$.

We call $B \subset \overline{\mathbb{R}}^{X}$ a function vector lattice if it is a real linear space under pointwise $=,+, s$, such that $h \in B$ implies $|h| \in B$; then $h \wedge k, h \vee k \in B$ for $h, k \in B$, thus $h \cap|k| \in B$.

\section{$I \mid B$ Loomis system (on $X$ ) means}

(9) $B$ is a function vector lattice, $I: B \rightarrow R$ is linear, $I(h) \geqslant 0$ if $h \in+B$.

$q B I$ means $q$ is an integral metric on $X$, (9) holds, $I$ is $q$-continuous in 0

If only an integral metric $q$ on $X$ and a function vector lattice $B$ are given, $q B 0$ is true with $I \equiv 0$, all the following results hold then.

THEOREM 1. (Aumann). If $q B I$ of (10) holds, then the set $B^{q}$ of $q$-integrable functions is closed with respect to $\pm, s \cdot(s \in \mathbb{R}), \|, \vee, \wedge, \cap$. Also $B \cup N_{q} \subset B^{q}=$ $\left(B^{q}\right)^{q}$ and there is a unique $q$-continuous extension $I^{q}: B^{q} \rightarrow \mathbb{R}$ of $I \mid B$.

For this $I^{q}$ one has $I^{q}(s f)=s I^{q}(f), I^{q}(f+g)=I^{q}(f)+I^{q}(g),\left|I^{q}(f)\right| \leqslant$ $I^{q}(|f|)=:\|f\|_{I^{q}}, I^{q}(k) \leqslant I^{q}(l)$ if $f, g, k, l \in B^{q}, s \in \mathbb{R}, k \leqslant l ;\|\|_{I^{q}}$ is a seminorm on $B^{q}$ which is $q$-continuous, so $q(|k|)=0$ implies $k \in B^{q}$ and $I^{q}(k)=0$.

Definition 3: If (5) holds, for $k, l \in \overline{\mathbb{R}}^{X}$, with $k^{+}:=k \vee 0$ :

$$
k=l(q) \text { respectively } k \leqslant l(q) \text { means } q(|k-l|)=0 \text { respectively } q\left((k-l)^{+}\right)=0 \text {. }
$$

Lemma 2. If (5) holds and $q$ is an integral metric on $X$, then $=(q)$ is an equivalence relation in $\overline{\mathbb{R}}^{X} ; \leqslant(q)$ is there transitive; they are further compatible with + of (1) and $s \cdot, s \in \mathbb{R}$ respectively $+\mathbb{R}$.

Corollary I. If $q B I$ of (10) holds, $f \in B^{q}, k \in \overline{\mathbb{R}}^{X}$ with $k=f(q)$ then $k \in B^{q}$ and $I^{q}(k)=I^{q}(f)$.

Corollary II. $q B I, f \in B^{q}$ imply $f_{e} \in B^{q}, f_{ \pm \infty}$ and $f_{u} \in N_{q}, I^{q}(f)=$ $I^{q}\left(f_{e}\right)$.

Here $k_{e}(x):=k(x)$ if $k(x) \in \mathbb{R},:=0$ else; $k_{u}:=k-k_{e}, k_{\infty}:=k_{u} \vee 0, k_{-\infty}:=$ $k_{u} \wedge 0$.

Corollary III. $q B I, f, g \in B^{q}, k \in \overline{\mathbb{R}}^{X}$ with $k(x)=f(x)+g(x)$ if $f(x) \in \mathbb{R}$ and $g(x) \in \mathbb{R}$ imply $k \in B^{q}$ and $I^{q}(k)=I^{q}(f)+I^{q}(g)$. 
Corollary IV. $q B I, f, g \in B^{q}$ imply $f \dot{ \pm} g, f \pm g \in B^{q}$ and $I^{q}(f \dot{ \pm} g)=$ $I^{q}(f) \pm I^{q}(g)$.

Corollary V. $q B I, f, g \in B^{q} f \leqslant g(q)$ imply $I^{q}(f) \leqslant I^{q}(g)$.

Corollary VI. The extension process $I \mid B \rightarrow I^{q} B^{q}$ is iteration complete.

This means: If $q B I$ holds, $\widetilde{B}:=B^{q} \cap \mathbb{R}^{X}, \widetilde{I}:=I^{q} \mid \widetilde{B}$, then $q \widetilde{B} \widetilde{I}$ holds, $\widetilde{B}^{q}=B^{q}$ and $\widetilde{I}^{q}=I^{q}$.

In the proofs one uses $\left|f_{e}-h_{n}\right| \leqslant\left|f-h_{n}\right|,\left|f_{u}\right| \leqslant 2\left|f-h_{n}\right|$, for Corollary III $|k-(h+l)| \leqslant|f-h|+|g-l|$, and, valid for any $a, b, c, d \in \overline{\mathbb{R}}, t \in+\overline{\mathbb{R}}$,

$$
\begin{aligned}
(a-b) \vee t & \leqslant(a-c) \vee t+(c-b) \vee t \\
((a+b)-(c+d)) & \vee t \leqslant((a-c) \vee t)+((b-d) \vee t) \\
a & \leqslant b+(a-b)^{+}
\end{aligned}
$$

Definition 4: With (5), $k \in \overline{\mathbb{R}}^{X}: k q-B$-measurable means that $k \cap h \in B^{q}$ for all $h \in+B$,

$$
M_{\cap}(q, B):=\left\{k \in \overline{\mathbb{R}}^{X}: k q B \text {-measurable }\right\}
$$

Theorem 2. If $q B I$ holds and $k \in \overline{\mathbb{R}}^{X}$, then the following three statements are equivalent:

(a) $k \in B^{q}$

(b) $k$ is $q$ - $B$-measurable and $|k| \in B^{q}$

(c) $k$ is $q$ - $B$-measurable and there is $\varphi \in B^{q}$ with $|k| \leqslant \varphi(q)$.

Proof: (a) $\Rightarrow$ (b) $\Rightarrow$ (c) by Theorem 1. For (c) $\Rightarrow$ (a), with (13) and Corollary IV we can assume $|k| \leqslant \varphi$; then $0 \leqslant \varphi$ and for $\varepsilon>0$ there is $h \in B$ with $q(|\varphi-h|)<\varepsilon$; since

$$
|\varphi-| h|| \leqslant|\varphi-h|, \quad|h| \epsilon+B \text {, one can assume } h \geqslant 0 \text {. }
$$

Then $|k-k \cap h|=|k \cap \varphi-k \cap h| \leqslant 2|\varphi-h|$ by (2), so $q(|k-k \cap h|)<2 \varepsilon$, or $k \in\left(B^{q}\right)^{q}=B^{q}$.

For convergence theorems, we need an analogue to "convergence in measure":

Definition 5: For $J$ net, $k, k_{j} \in \mathbb{R}^{X}$ for $j \in J,(5)$ :

$$
k_{j} \rightarrow k(q, B) \quad \text { means }
$$

for each $\varepsilon>0$ and $h \in+B$ there is $j=j_{\epsilon, h} \in J$ such that $q\left(\left|k-k_{i}\right| \wedge h\right)<\varepsilon$ if $j \leqslant i \in J$ ( $q$-B-local convergence).

If with integral metric $q$, fixed $B$ and the same $J, k_{j} \rightarrow k(q, B)$ and $l_{j} \rightarrow l(q, B)$, then $s k_{j} \rightarrow s k(q, B)$ and $k_{j}+l_{j} \rightarrow k+l(q, B)$ for $s \in \mathbb{R}$ by (8), (3). 
THEOREM 3. If $q$ is an integral metric on $X, 0 \in B \subset \overline{\mathbb{R}}^{X}, J$ net, $k_{j}, k \in$ $\overline{\mathbb{R}}^{X}$ with $k_{j} \rightarrow k(q, B)$ and $\left|k_{j}-k\right| \leqslant \varphi(q)$ for $j \in J$ with some $\varphi \in B^{q}$, then $q\left(\left|k-k_{j}\right|\right) \rightarrow 0$.

PROOF: If $l_{j}:=\left|k_{j}-k\right| \leqslant \varphi$, then as in the proof of Theorem 2 , for $\varepsilon>0$ choose $h \in+B$ with $q(|\varphi-h|)<\varepsilon$, then $j$ with $q\left(\left|k-k_{i}\right| \wedge h\right)<\varepsilon$ if $i \geqslant j$. Now $l_{i}=l_{i} \wedge h+\left(l_{i}-l_{i} \wedge h\right)=l_{i} \wedge h+\left(l_{i} \wedge \varphi-l_{i} \wedge h\right) \leqslant l_{i} \wedge h+|\varphi-h|$, so $q\left(l_{i}\right)<2 \varepsilon$ if $i \geqslant j=j_{e}$.

In the general case, $l_{i} \leqslant \varphi+p_{i} \leqslant|\varphi|+p_{i}$ by (15) with $p_{i}:=\left(l_{i}-\varphi\right)^{+} \in N_{q} ;$ then $0 \leqslant h_{i}:=l_{i}-l_{i} \wedge p_{i} \leqslant|\varphi| \in B^{q}$ for $i \in J$ and $q\left(h_{i} \wedge h\right) \leqslant q\left(l_{i} \wedge h\right) \rightarrow 0$ if $h \in+B$, that is $h_{i} \rightarrow 0(q, B)$, so $q\left(h_{i}\right) \rightarrow 0$ by the above. Now $l_{i}=h_{i}+l_{i} \wedge p_{i}$, therefore $0 \leqslant q\left(l_{i}\right) \leqslant q\left(h_{i}\right)+q\left(p_{i}\right)=q\left(h_{i}\right) \rightarrow 0$.

Corollary VII. (Lebesgue's convergence theorem for $B^{q}$ ): If $q B I$ holds, $f_{j}$, $\varphi \in B^{q}, k \in \overline{\mathbb{R}}^{X}, f_{j} \rightarrow k(q, B)$ and $\left|f_{j}-k\right| \leqslant \varphi(q)$ for $j \in J$ then $k \in B^{q}$, $q\left(\left|f_{j}-k\right|\right) \rightarrow 0, I^{q}\left(f_{j}\right) \rightarrow I^{q}(k)$.

Proof: By Theorem 3 and $1, k \in\left(B^{q}\right)^{q}=B^{q}$ and $\left|I^{q}\left(f_{j}\right)-I^{q}(k)\right| \leqslant I^{q}\left(\left|f_{j}-k\right|\right)$ $\rightarrow 0$.

Lemma 3. If (5) holds and $q$ is an integral metric on $X, g_{j} \in M_{\cap}(q, B), k \in \overline{\mathbb{R}}^{X}$ and $g_{j} \rightarrow k(q, B)$ then $k \in M_{\cap}(q, B)$.

ProOF: $\left|g_{j} \cap h-k \cap h\right| \leqslant 2\left(\left|g_{j}-k\right| \wedge h\right)$ by $(2)$, so $k \cap h \in\left(B^{q}\right)^{q}=B^{q}$ by Lemma 1.

Lemma 4. If $q B 0$ of (10) holds, $f, g \in M_{\cap}(q, B), l \in B^{q}, s \in \mathbb{R}$ and $k \in \overline{\mathbb{R}}^{X}$ then $l,|f|, f \wedge g, f \vee g, f^{ \pm}, s f, f \cap|g|, f+l$ belong to $M_{\cap}(q, B)$.

$f+g \in M_{\cap}(q, B)$ if there are $f_{0}, g_{0} \in B^{q}$ with $f \geqslant f_{0}$ and $g \geqslant g_{0}$.

Besides $k \in M_{\cap}(q, B) \Leftrightarrow k \wedge h \in M_{\cap}(q, B)$ for $h \in+B \Leftrightarrow f^{+}, f^{-} \in M_{\cap}(q, B)$.

With only $f \geqslant 0$, for example, in general $f+g \notin M_{n}(q, B)$. ([10], A5.100).

PRoOF: $|r| \cap t=|r \cap t|,(-r) \cap t=-(r \cap t),(s r) \cap(s t)=s(r \cap t),(p \wedge r) \cap t=$ $(p \cap t) \wedge(r \cap t), r^{ \pm}=( \pm r) \vee 0,(p \cap|r|) \cap t=(p \cap t) \cap|r \cap t|$ if $p, r, s, t \in \mathbb{R}, s \geqslant 0$, $t \geqslant 0,(2),(3)$ and Theorem 1. $f+l \in M_{\cap}$ by Lemma 3 if $f+v \in M_{\cap}$ for $v \in B$; but then $(f+v) \cap h=\left(\left(f_{v}\right) \wedge h\right) \vee(-h)=(((f \wedge(h-v))+v) \vee(-h))=[(f \wedge(h-v)) \vee$ $(-h-v)]+v ;[] \in M_{\cap}$ since $M_{\cap}$ is $\wedge$ and $\vee$-closed; also $\|[\| \leqslant|h|+|v| \in B$, do [ ] $\in B^{q}$ by Theorem 2, then $(f+v) \cap h \in B^{q} . k \cap h=(k \wedge h) \cap h=k^{+} \wedge h-k^{-} \wedge h$ if $h \in+B$, Theorems 1,2 . If $f, g \geqslant 0$, then $(f+g) \cap h=(f \wedge h+g \wedge h) \wedge h \in B^{q}$; else $f+g=\left(f^{+}+g^{+}\right)-\left(f^{-}+g^{-}\right)$, with $f^{+}+g^{+} \in M_{n},\left|f^{-}+g^{-}\right| \leqslant\left|f_{0}\right|+\left|g_{0}\right| \in B^{q}$, so $-l:=f^{-}+g^{-} \pi B^{q}$ by Theorem 2 .

Corollary VIII. If $q B I$ holds, $g_{j} \in M_{\cap}(q, B), k \in \overline{\mathbb{R}}^{X}, g_{j} \rightarrow k(q, B)$ and there is $\varphi \in B^{q}$ with $|k| \leqslant \varphi(q)$, then $k \in B^{q}$. 
This variant to Corollary VII follows from Lemma 3 and Theorem 2; however, even if $g_{n} \in B^{q}, I^{q}\left(g_{n}\right) \rightarrow I^{q}(k)$ is false in general.

Also Theorem 3/Corollary VII, VIII become false if only $\left|f_{j}-k\right| \leqslant \varphi\left(q_{B}\right)$ with $q_{B}$ of Section 2 (Example 2 in Günzler [11, see Section 2]).

Finally, $\mathfrak{L}^{q}:=B^{q} / N_{q}$ is a vector lattice with integral $I^{q}$ and norm $I^{q}(|\cdot|)$ (Au$\operatorname{mann}[1, \mathrm{p} .445])$.

\section{LOCAL INTEGRAL METRICS}

DEFinition 6: If $X, B, q$ are as in (5), then

$$
q_{B}(k):=\sup \{q(k \wedge h) ; h \in+B\}, \text { for } k \in+\overline{\mathbb{R}}^{X} \text {. }
$$

This is a simplified version of Schäfke's definition [19, p.120]. Here it gives all the relevant results, under weaker assumptions.

LEmMA 5. If (5) holds and $q$ is an integral metric on $X$, then $q_{B}$ of (16) is also an integral metric on $X$, with $q_{B B}=q_{B} \leqslant q ; q_{B}(k)=q(k)$ if $k \in+\overline{\mathbb{R}}^{X}$ and $k \leqslant$ some $h \in B$, so $k_{j} \rightarrow k(q, B)$ of Definition 5 is equivalent with $k_{j} \rightarrow k\left(q_{B}, B\right)$; if additionally $|B| \subset B$, then $q_{B}(k \wedge \varphi)=q(k \wedge \varphi)$ for $k \in+\overline{\mathbb{R}}^{X}, \varphi \in+B^{q}$.

ProOF: As in Schäfke [19, p.120-121], with

$$
(r+s) \wedge t \leqslant r \wedge t+s \wedge t \quad \text { if } r, s, t \in+\overline{\mathbb{R}}^{X} \text {. }
$$

LEMMA 6. If $q B I$ of (10) holds, then also $q_{B} B I$ is true, so $B^{q_{B}}$ and $I^{I_{B}}: B^{q_{B}} \rightarrow$ $\mathbb{R}$ are well defined, with

$$
B \subset B^{q} \subset B^{q_{B}}, \quad I \subset I^{q} \subset I^{q_{B}}
$$

TheOREM 4. If (5) holds, $q$ is an integral metric on $X, k_{j}, k \in \overline{\mathbb{R}}^{X}$, then $q_{B}\left(\left|k_{j}-k\right|\right) \rightarrow 0$ if and only if

$$
k_{j} \rightarrow k(q, B) \quad \text { and } \quad\left(k_{j}\right) \text { is a } q_{B} \text {-Cauchy-net. }
$$

ProOF: As in Schäfke [19, p.121-122].

Corollary IX. If (5) holds with integral metric $q$, then $f \in B^{q_{B}}$ if and only if to $f$ there exists a sequence $\left(h_{n}\right)$ with $h_{n} \in B$ and

$$
h_{n} \rightarrow f(q, B), \quad q\left(\left|h_{n}-h_{m}\right|\right) \rightarrow 0 \quad \text { as } n, m \rightarrow \infty
$$


Corollary X. If (5) holds with integral metric $q, f_{j} \in B^{q_{B}}, k \in \overline{\mathbb{R}}^{X}, f_{j} \rightarrow k$ $(q, B)$ and $\left(f_{j}\right) q_{B}$-Cauchy then $k \in B^{q_{B}}, q_{B}\left(\left|k-f_{j}\right|\right) \rightarrow 0$ (and $I^{q_{B}}\left(f_{j}\right) \rightarrow I^{q_{B}}(k)$ if $q B I$ holds).

This closedness-property of $B^{q_{B}}$ can be looked at as a convergence theorem for $B^{q_{B}}$; by Example 2 of [11] an analogue for general $B^{q}$ is false.

COROLlary XI. If $q B I$ holds, for $f \in \overline{\mathbb{R}}^{X}$ the following statements are equivalent:

(a) $f \in B^{q}$

(b) $f \in B^{q_{B}}$ and there exists $\varphi \in B^{q}$ with $|f| \leqslant \varphi(q)$

(c) $f \in M_{\cap}\left(q_{B}, B\right)$ and there exists $\varphi \in B^{q}$ with $|f| \leqslant \varphi(q)$.

So $M_{\cap}\left(q_{B}, B\right)=M_{\cap}(q, B)$.

Proof: (a) $\Rightarrow$ (b) with $\varphi=|f|$ and (b) $\Rightarrow$ (c) follows from Theorems 1 and 2.

For (c) $\Rightarrow$ (a), $f \cap h \in B^{q_{B}}$ for $h \in+B$, so there are $h_{n} \in B$ with $q_{B}\left(\left|f \cap h-h_{n} \cap h\right|\right) \leqslant q_{B}\left(2\left|f-h_{n}\right|\right) \rightarrow 0$ by (2); since $\left|f \cap h-h_{n} \cap h\right| \leqslant 2 h \in B$, by Lemma $5 q\left(\left|f \cap h-h_{n} \cap h\right|\right)=q_{B}\left(\left|f \cap h-h_{n} \cap h\right|\right) \rightarrow 0$, thus $f \cap h \in B^{q}$; Theorem 2 yields $f \in B^{q}$. $|f \cap h| \leqslant h$ for the $M_{n}$-statement.

Again, $|f| \leqslant \varphi(q)$ cannot be weakened here to $\leqslant\left(q_{B}\right)$; similarly $=(q)$ is stronger than $=\left(q_{B}\right)$. One only has, for $k, l \in \overline{\mathbb{R}}^{X}$, with $(5)$ :

$$
k=l\left(q_{B}\right) \Leftrightarrow k=l(q, B): \Leftrightarrow K_{n}:=k \rightarrow l(q, B) \text { (Definition 5), }
$$

similarly for $\leqslant\left(q_{b}\right)$.

For $q_{B}$ Theorem 2 can be sharpened with:

Definition 7: (Schäfke). Under (5), $q$ is called $B$-semiadditive if one has

$$
h_{n} \in+B, \sup \left\{q\left(\sum_{1}^{n} h_{m}\right) ; n \in \mathbb{N}\right\}<\infty \Rightarrow q\left(h_{n}\right) \rightarrow 0 \text { as } n \rightarrow \infty \text {. }
$$

$q$ is called $B$-additive if $h, k \in+B$ imply $q(k+h)=q(k)+q(h)$.

Obviously " $q B$-additive" implies " $q B$-semiadditive" if $+B$ is + closed.

Any integral metric extension of \|\|$_{p}=\left(\int||^{p} d \mu\right)^{1 / p}$ from $L^{p}(\mu, \bar{R})$ is $B$ semiadditive if $1 \leqslant p<\infty$, where $B=$ step functions $B_{\Omega}$ or $L^{p}$, with arbitrary measure space $(X, \Omega, \mu)$; only for $p=1$ has one $B$-additivity; see Section 3.F.

LEMma 7. If (5) holds, $q$ is an integral metric on $X,|B|+|B| \subset|B| \subset B$ and $q B$-semiadditive then $q$ is $B^{q}$-semiadditive and $q_{B} B^{q_{B}}$-semiadditive.

This analogue to Satz 5.1 of Schäfke [19] follows as in [16, p.161-162]. 
Theorem 5. If $q B 0$ of (10) holds, $q$ is $B$-semiadditive, $k$ is $q$-B-measurable and $q_{B}(|k|)<\infty$, then $k \in B^{q_{B}}$.

Proof: With Lemmas 5, 4 and Theorem 2 one can assume $k \geqslant 0$; then one can argue as in Schäfke [19, p.127], showing first: For $\varepsilon>0$ there exists $h_{\varepsilon} \epsilon+B$ with

$$
q\left(k \wedge h-k \wedge h_{\varepsilon}\right)<\varepsilon \quad \text { for all } h \text { with } h_{\varepsilon} \leqslant h \in B
$$

Without " $B$-semiadditive" or with $q$ instead of $q_{B}$ Theorem 5 becomes false though $q$ - and $q_{B}$-measurability coincide (Corollary XI): Example 1 below.

LemmA 8. If (5) holds, $k, k_{j} \in+\overline{\mathbb{R}}^{X}, k_{j} \rightarrow k(q, B)$ then $q_{B}(k) \leqslant \underline{\operatorname{Lim}} q_{B}\left(k_{j}\right)$.

Proof: $q\left(\left|k \wedge h-k_{j} \wedge h\right|\right) \leqslant 2 q\left(\left|k-k_{j}\right| \wedge h\right) \rightarrow 0$ by (2) if $h \in+B$; if $a<q_{B}(k)$ there is $h \in+B$ with $a<q(k \wedge h)$; now $q(k \wedge h)-q\left(k_{j} \wedge h\right) \leqslant q\left(\left|k \wedge h-k_{j} \wedge h\right|\right) \rightarrow 0$, so $a<q\left(k_{j} \wedge h\right) \leqslant q_{B}\left(k_{j}\right)$ if $j \geqslant j_{a, h}$.

With this and Lemma 3 one gets the following analogue to Fatou's Lemma.

Corollary XII. If $q B I$ holds, $q$ is $B$-semiadditive, $f_{j} \in M_{\cap}(q, B), k \in \overline{\mathbb{R}}^{X}$, $f_{j} \rightarrow k(q, B)$ and $\underline{\operatorname{Lim}} q_{B}\left(\left|f_{j}\right|\right)<\infty$ or $q_{B}(|k|)<\infty$, then $k \in B^{q_{B}}$.

Here the same remarks as after Theorem 5 apply; also in general $q_{B}\left(\left|f_{j}\right|\right) \nrightarrow q_{B}(|k|)$.

Theorem 6. (Monotone Convergence Theorem for $B^{q_{B}}$ )

Assume $q B I, q B$-semiadditive, $k \in \overline{\mathbb{R}}^{X},\left(f_{j}\right)_{j \in J}$ net with $f_{j} \in B^{\boldsymbol{q}_{B}}, f_{j} \rightarrow$ $k(q, B), f_{i} \leqslant f_{j}\left(q_{B}\right)$ if $i \leqslant j \leqslant J$, and $\underline{\operatorname{Lim}}_{j} q_{B}\left(\left|f_{j}-f_{j_{0}}\right|\right)<\infty$ for some $j_{0} \in J$. Then $k \in B^{q_{B}}, q_{B}\left(\left|f_{j}-k\right|\right) \rightarrow 0, f_{j} \leqslant k\left(q_{B}\right), I^{q_{B}}\left(f_{j}\right) \rightarrow I^{q_{B}}(k)=\sup _{j \in J} I^{q_{B}}\left(f_{j}\right)$.

EXAMPLE 1: If $X=\mathbb{N}, B=c_{00}:=\left\{a \in \mathbb{R}^{\mathbf{N}}: a(n)=0\right.$ for $n \geqslant$ some $\left.n_{a}\right\}$, $q(k)=q_{B}(k)=\|k\|_{\infty}:=\sup _{n \in \mathbb{N}}|k(n)|, f_{n}(m):=1$ if $1 \leqslant m \leqslant n,:=0$ else, $I=0$, then one has $q B I, 0 \leqslant f_{n-1} \leqslant f_{n} \rightarrow k:=1(q, B), q_{B}\left(f_{n}\right) \equiv 1$, but $k \notin B^{q}=B^{q_{B}}=$ $c_{0}(\mathbb{N}, \mathbb{R}) \subset \mathbb{R}^{\mathbf{N}}$, so $q$ is not $B$-semiadditive.

If $q(h)<\infty$ for $h \in+B$, also $q_{B} \neq \infty$ on $B^{q_{B}}$, the $\underline{\lim } q_{B}\left(\left|f_{j}-f_{j_{0}}\right|\right)<\infty$ if and only if $\underline{\lim } q_{B}\left(\left|f_{j}\right|\right)<\infty$.

For $B^{q}$ instead of $B^{q_{B}}$ Theorem 6 becomes false by Example 2 of [11].

Proof of Theorem 6: $g_{j}:=f_{j}-f_{j_{0}} \rightarrow l:=k-f_{j_{0}}(q, B)$ by the remark after Definition 5; Lemma 4, Corollary XII and III give $l, k \in B^{q_{B}}$.

Furthermore $f_{i}-f_{j} \rightarrow f_{i}-k(q, B)$ and $p_{i j}:=\left(f_{i}-f_{j}\right)^{+} \rightarrow\left(f_{i}-k\right)^{+}(q, B)$ as $j \rightarrow \infty$ by (2); since $p_{i j} \in+N_{q_{B}}$ if $i \leqslant j$, one gets $\left(f_{i}-k\right)^{+} \in N_{q_{B}}$ or $f_{i} \leqslant k\left(q_{B}\right)$ for $i \in J$ by Lemma 8; Corollary V yields $I^{q_{B}}\left(f_{i}\right) \leqslant I^{q_{B}}(k)$. 
With (13) and $p_{i}:=p_{j_{0} i}$ one has $0 \leqslant g_{i}+p_{i}$, furthermore one can show $g_{i} \leqslant$ $g_{j}+p_{i j} \in N_{q_{B}}$, yielding $q_{B}\left(\left|g_{i}\right|\right) \leqslant q_{B}\left(\left|g_{j}\right|\right)$ or $\sup _{j \geqslant j_{0}} q_{B}\left(\left|g_{j}\right|\right)=\underline{\lim } q_{B}\left(\left|g_{j}\right|\right):=t_{0}<\infty$.

$\left(f_{j}\right)$ is $q_{B}$-Cauchy, if for each $\varepsilon>0$ there is $i_{\varepsilon}$ with $q_{B}\left(f_{j}-f_{i_{\varepsilon}}\right) \leqslant \varepsilon$ if $j \geqslant i_{\varepsilon}$; if this is false, there is $\varepsilon_{0}>0$ and recursively a sequence $\left(j_{m}\right)$ with $j_{0} \leqslant j_{1} \leqslant \ldots$ and $q_{B}\left(\left|f_{j_{k}}-f_{j_{k-1}}\right|\right)>\varepsilon_{0}, k \in \mathbb{N}$. Since $\left|f_{j}-f_{i}\right| \leqslant\left(f_{j}-f_{i}\right)+2 p_{i j}$, inductively $\sum_{1}^{n}\left|f_{j_{k}}-f_{j_{k-1}}\right| \leqslant\left|f_{j_{n}}-f_{j_{0}}\right|+l_{n}$ with $q_{B}\left(l_{n}\right)=0$. The associativity of $\dot{+}$ gives

$$
\begin{aligned}
\sum_{1}^{n+1}\left|f_{j_{k}}-f_{j_{k-1}}\right| & \leqslant\left(\left|f_{j_{n}}-f_{j_{0}}\right|+l_{n}\right)+\left(\left(f_{j_{n+1}}-f_{j_{n}}\right)+r_{1}\right) \\
& \leqslant\left(\left(f_{j_{n}}-f_{j_{0}}\right)+r_{2}\right)+\left(\left(f_{j_{n+1}}-f_{j_{n}}\right)+r_{1}\right) \\
& =\left[\left(f_{j_{n+1}}-f_{j_{n}}\right)+\left(f_{j_{n}}-f_{j_{0}}\right)\right]+r_{3} \\
& \leqslant\left|f_{j_{n+1}}-f_{j_{0}}\right|+r_{4}
\end{aligned}
$$

with $r_{1}, \ldots, r_{4} \in N_{q_{B}}$, since with (3) one can show $[\cdots] \leqslant\left|f_{j_{n+1}}-f_{j_{0}}\right|+p_{j_{0}, j_{n}}+$ $p_{j_{n}, j_{n+1}}$.

But then $q_{B}\left(\sum_{1}^{n}\left|f_{j_{k}}-f_{j_{k-1}}\right|\right) \leqslant q_{B}\left(\left|f_{j_{n}}-f_{j_{0}}\right|\right) \leqslant t_{0}<\infty$ for $n \in \mathbb{N}$. With Lemma 7 one gets the contradiction $q_{B}\left(\left|f_{j_{k}}-f_{j_{k-1}}\right|\right) \rightarrow 0$.

Theorem 4 gives therefore $q_{B}\left(\left|f_{j}-k\right|\right) \rightarrow 0$, then $I^{q_{B}}\left(f_{j}\right) \rightarrow I^{q_{B}}(k)$.

Lemma 9. If $0 \in|B| \subset B \subset \overline{\mathbb{R}}^{X}, q$ is an integral metric on $X, \tilde{q}:=q_{B}$ and $B \subset \widetilde{B} \subset B^{q}$, then $\widetilde{q}_{B}=\widetilde{q}_{\widetilde{B}}=\tilde{q}_{B} \leqslant q$ and all are integral metrics, $B^{q} \subset B^{q} \widetilde{B} \subset \widetilde{B}^{q} \widetilde{B} \subset$ $B^{q}=\widetilde{B}^{q}$.

This follows from Lemma $5,(7),(2)$ and the definitions.

EXAMPLE 2: $X=\mathbb{N}, B=c_{00}$ as in Example $1, q:=0$ on $c_{00}, q:=1$ else, $q$ is even $B$-additive, $\widetilde{B}:=B^{q_{B}} \cap \mathbb{R}^{X}$; here all five " $C$ " and the two " $\leqslant$ " in Lemma 9 are strict.

Especially, the extension process $I\left|B \rightarrow I^{q_{B}}\right| B^{q_{B}}$ is also iteration complete (see Corollary VI):

Corollary XIII. If $q B I$ holds and $\widetilde{B}=B^{q_{B}} \cap \mathbb{R}^{X}, \tilde{I}:=I^{q_{B}} \mid \widetilde{B}, \tilde{q}:=q_{B}$, then $q \widetilde{B} \tilde{I}$ and $\tilde{q} \widetilde{B} \widetilde{I}$ hold, $B^{q_{B}}=B^{\tilde{q}}=B^{\tilde{q}} \widetilde{B}=\widetilde{B}^{\tilde{q}}=\widetilde{B}^{\tilde{q}_{B}}=\widetilde{B}^{\tilde{q}}, I^{q_{B}}=\widetilde{I}^{q_{B}}=\widetilde{I}^{q_{B}}$.

Definition 8: For $M \subset \overline{\mathbb{R}}^{X}, q:+\overline{\mathbb{R}}^{X} \rightarrow+\overline{\mathbb{R}}: C_{\infty}(q, M)$ means

$$
q(h-h \wedge n) \rightarrow 0 \text { as } n \rightarrow \infty, \text { for each } h \in+M \text {. }
$$

$M$ is called Stonean if $h \wedge 1 \in M$ for each $h \in+M$.

With this, $B^{q_{B}}$ is closed with respect to improper integration: 
Corollary XIV. Assume $q B 0$ of (10), $C_{\infty}(q, B), q B$-semiadditive and $k \in$ $\overline{\mathbb{R}}^{X}$; if $k \cap n$ is $q_{B}-B$-measurable for each $n \in \mathbb{N}$ and $\sup _{n \in \mathbb{N}}(|k \cap n|)<\infty$, then $k \in B^{q_{B}}$ and $q_{B}(|k-k \cap n|) \rightarrow 0$.

Proof: If $h \in+B, k \cap(h \wedge n)=(k \cap n) \cap h \in B^{q}$ by Corollary XI and $q(|k \cap h-k \cap(h \wedge n)|) \leqslant 2 q(h-h \wedge n) \leqslant q(|k| \cap h-|k| \cap(h \wedge n))+q(|k| \cap(h \wedge n)) \leqslant$ $2 q(h-h \wedge n)+q_{B}(|k| \wedge n) \leqslant 1+\sup _{m} q_{B}(|k \cap m|)$ for some $n=n(h)$, Theorem 5 yields $k \in B^{q_{B}}$.

For $\varepsilon>0$ there is $h \in B$ with $q_{B}(|k-h|)<\varepsilon$, then $q_{B}(|k-k \cap n|) \leqslant$ $q_{B}(|k-h|)+q_{B}(|h-h \cap n|)+q_{B}(|h \cap n-k \cap n|) \leqslant \varepsilon+q_{B}(|h|-|h| \wedge n)+2 \varepsilon=$ $3 \varepsilon+q(|h|-|h| \wedge n)$ by Lemma 5 , so $\leqslant 4 \varepsilon$ for $n>$ some $n_{\varepsilon}$.

Especially we have $k \cap n \rightarrow k(q, B)$; for this " $q B$-semiadditive" and " $k \cap n q_{B}$ - $B$ measurable" are superfluous, provided $k \in B^{q_{B}}$.

" $k \cap n q_{B}-B$-measurable" is also necessary for Stonean $B$, since then $B^{p}$ is Stonean for an integral metric $p$, implying $f \cap t \in B^{p}$ if $f \in B^{p}, t \in+\mathbb{R}$.

Again all assumptions are independent and essential, Corollary XIV also becomes false if one replaces $q_{B}$ by $q$.

For Stonean $B$ the $C_{\infty}$-continuity condition can be weakened in Corollary XIV:

LEmma 10. If $M \subset+\overline{\mathbb{R}}^{X}$ with $h \wedge n$ and $h-h \wedge n \in M$ for $h \in M, n \in \mathbb{N}, q$ is an $M$-semiadditive integral metric with $q(h \wedge n) \rightarrow q(h)$ as $n \rightarrow \infty$ if $h \in M$, then $q(h-h \wedge n) \rightarrow 0$ as $n \rightarrow \infty$ for $h \in M$ with $q(h)<\infty$.

Proof: If $q(h-h \wedge n)>\varepsilon_{0}>0$ for $n \in \mathbb{N}$, there are $n_{1}<n_{2}<\cdots \in \mathbb{N}$ with $q\left(h \wedge n_{j+1}-h \wedge n_{j}\right)>\varepsilon_{0}$ for $j \in \mathbb{N}$, since $(h-h \wedge n) \wedge m=h \wedge(n+m)-h \wedge n$; with $k j:=h \wedge n_{j+1}-h \wedge n_{j} \in M$ one has $q\left(\sum_{i}^{m} k_{j}\right)=q\left(h \wedge n_{m+1}-h \wedge n_{1}\right) \leqslant q(h)$, yielding the contradiction $q\left(k_{j}\right) \rightarrow 0 .(q(h)<\infty$ is essential here.)

\section{APPLICATIONS AND EXAMPLES}

Definition 9: For $\emptyset \neq M \subset P \subset \overline{\mathbb{R}}^{X}, T: P \rightarrow \overline{\mathbb{R}}$ :

$$
T^{M}(k):=\operatorname{Inf}\{T(h): k \leqslant h \in M\} \text { for } k \in \overline{\mathbb{R}}^{X}, \text { with } \inf \emptyset=\infty
$$

LEMma 11. If $0 \in M=M+M \subset \overline{\mathbb{R}}^{X}, T: M \rightarrow \overline{\mathbb{R}}$ with

$$
T(h) \leqslant T(k) \dot{+}(l) \text { if } h \leqslant k \dot{+} l \text { and } h, k, l \in M,
$$

then $T^{M}: \overline{\mathbb{R}}^{X} \rightarrow \overline{\mathbb{R}}$ is well defined, $=T$ on $M$ and satisfies (24) with $h, k, l \in \overline{\mathbb{R}}^{X}$; $\left(T^{M}\right)^{M}=T^{M}$ on $\overline{\mathbb{R}}^{X}$. (Here + cannot be replaced by.+$)$. 
A, Proper Riemann integrals.

$$
\begin{aligned}
& \mu \mid \Omega \text { means } \Omega \text { is a semiring of sets from } X, \mu: \Omega \rightarrow[0, \infty) \\
& \text { is additive on } \Omega, B_{\Omega}:=\text { step functions } S(\Omega, \mathbb{R}),
\end{aligned}
$$$$
I_{\mu}:=\int \ldots d \mu \text { on } B_{\mathbf{\Omega}}[10, \mathrm{p} .10-13] \text {. }
$$

With $q=I_{\mu}^{-}:=\left(I_{\mu}\right)^{B_{n}}$ of Definition 9 , Aumann $[1$, p.447-448] respectively Lemma 11 give

$$
I_{\mu}^{-} B_{\Omega} I_{\mu} \text { of }(10) \text { holds, } I_{\mu}^{-} \text {is } B_{\Omega} \text {-additive and positive-homogeneous; }
$$

$$
R_{e}^{1}(\mu, \mathbb{R}):=\left(B_{\Omega}\right)^{I_{\mu}^{-}}, I_{\mu}: R_{e}^{1}(\mu, \mathbb{R}) \rightarrow \mathbb{R} \text { (abstract proper }
$$

Riemann- $\mu$-integral) are well defined, $I_{\mu}:=\left(I_{\mu}\right)^{I_{\mu}^{-}}$of Theorem 1.

$$
X=\mathbb{R}, \Omega=\{[a, b):-\infty<a \leqslant b<\infty\}, \mu([a, b))=b-a \text { give the classical }
$$
proper Riemann integrable functions, as for $\mathbb{R}^{n}[10, \mathrm{p} .216]$.

B, abstract Riemann- $\mu$-integration. With (25), $I\left|B=I_{\mu}\right| B_{\Omega}$ and the $B_{\Omega}$ additive integral metric $q=I_{\mu}^{-}$of $A$, for

$$
R_{1}(\mu, \overline{\mathbb{R}}):=B^{q_{B}}, \int \ldots d \mu:=\left(I_{\mu}\right)^{q_{B}}
$$

all results of Sections 1-2 are applicable, $B_{\Omega}$ is Stonean $C_{\infty}\left(I_{\mu}^{-}, B_{\Omega}\right)$ holds, $R_{e}^{\mathbf{1}}(\mu, \mathbb{R}) \subset$ $R_{1}(\mu, \overline{\mathbb{R}}) \cap \mathbb{R}^{X}=R_{1}(\mu, \mathbb{R})$ of $[10$, p.70-144] $=L(X, \Omega, \mu, \mathbb{R})$ of Dunford-Schwartz $\left[8\right.$, p.112] if $X \in \Omega . k_{j} \rightarrow k(q, B) \Leftrightarrow k_{j} \rightarrow k \mu$-locally, $M_{\cap}(q, B)=M R[\mu, \overline{\mathbb{R}}][11$, Lemma 9], [10, p.142]. Our convergence theorems are still generalisations of those for $R_{1}(\mu, \mathbb{R})$, Corollary XIV is new even then.

C, Loomis completions. Given $I \mid B$ with (9), and $q:=I^{-} /+\overline{\mathbb{R}}^{X}$ one has:

$$
R_{1}:=R_{1}(I \mid B):=B^{q_{B}}, \quad J:=I^{q_{B}}: R_{1} \rightarrow \mathbb{R} \text {, where } I^{-}:=I^{B} \text { of }
$$

Definition 9 and $\left(I^{-}\right)_{B}$ of Definition 5 , are $B$-additive.

$R_{1}(B / I) \supset R_{\mathrm{e}}^{1}=$ two-sided completion $R$ of Loomis [13, p.170], $R_{1}=$ one-sided completion $U$ of Loomis [13, p.178], = I-integrable functions of [14] = closure of $B$ in $\overline{\mathbb{R}}^{X}$ with respect to the distance $d(k, l):=\left(I^{-}\right)_{B}(|k-l|) . A$ and $B$ are special cases. This extension process $I|B \rightarrow J| R_{1}(I \mid B)$ is by [14, p.115-119] iteration complete, that is $R_{1}(I \mid B)=R_{1}(J \mid \widetilde{B})$ with $\widetilde{B}:=R_{1}(I \mid B) \cap \mathbb{R}^{X}$. 
D, Finitely-additive Daniell extension. For $I \mid B$ with (9) it is introduced in [3], for $k \in \overline{\mathbb{R}}^{X}$,

$$
\begin{aligned}
& I^{+}(k):=\sup \{I(h): h \leqslant k, h \in B\}, \\
& B^{+}:=\left\{k \in \overline{\mathbb{R}}^{X}:-\infty<I^{+}(k), \quad k=\sup \{h: k \geqslant h \in B\}\right\}, \\
& B_{+}:=\left\{f \in B^{+}: I^{+}(f+g) \leqslant I^{+}(f)+I^{+}(g) \text { for } g \in B^{+}\right\} \\
& B \subset B_{(+)}:=\left\{f \in B_{+}: I^{+}(f)<\infty\right\} \subset B_{+} \subset B^{+}, \\
& \left.\bar{I}:=\left(I^{+}\right)^{B_{+}} \text {(Definition } 9\right), \text { a } B \text {-additive integral metric } q \\
& \quad \text { on }+\overline{\mathbb{R}}^{X}, \text { with } q B I, \\
& \bar{B}:=B^{q}=\text { summable functions } B_{0} \text { of }[3], I^{q}=\bar{I} \text { on } \bar{B} ;
\end{aligned}
$$

the convergence theorems of Section 1 are those of [11]; by [14, Theorem 6.4] one has

$$
R_{e}^{1}(I \mid B) \subset \bar{B}, \quad R_{1}(I \mid B) \subset \bar{B}+\left\{f \in R_{1}(I \mid B): R_{1}-I(|f|)=0\right\}
$$

In the classical case $R_{1}=L^{1}=L_{1}=\bar{B}$ by (43)-(44), but in general Section 2 is not applicable ([11, Example 2], $R_{1} \subset \bar{B}, \bar{B} \subset R_{1}$.

E, localisation of the Daniell-analogue.

$$
L:=L(I \mid B):=B^{q_{B}} \quad J:=I^{q_{B}}: L \rightarrow \mathbb{R}, \quad q=\bar{I} \mid+\overline{\mathbb{R}}^{X}
$$

with $B, I, \bar{I}$ of $D$ allow an application of all results of Sections $1-2 ;(18)$ and $\bar{I} \leqslant I^{B}$ imply

$$
\bar{B} \subset L, \quad R_{e}^{1} \subset R_{1}(I \mid B) \subset L, \text { with coinciding integrals, }
$$

all $\subset$ are strict $\left[11\right.$, Exercise 2.3], and we have a generalisation of [3]. With $C_{\infty}(B, I)$ of [11] one can show $1 \in \bar{B} \Rightarrow L=\bar{B} ; 1 \in L \Rightarrow L=\bar{B}+N_{\bar{I}_{B}} ;$ with $C_{\infty}: B_{(+)} \subset R_{1} \Leftrightarrow$ $R_{1} \subset \bar{B} \Leftrightarrow R_{1}=L$. See also (43)-(44).

One has always the iteration closures of Theorem 1 and Corollary XII, where $\widetilde{B}:=L(I / B) \cap \mathbb{R}^{X}$ and $\bar{I}:=J / \widetilde{B}$.

Additional properties of $L(B / I)$ will be treated elsewhere, see also [7].

F, $L^{p}$-SPACES. For $q:=\overline{\mathbb{R}}^{X} \rightarrow+\overline{\mathbb{R}}, 0<p$ real $<\infty$, with $k^{p}(t):=(k(t))^{p}, 0^{p}:=0$, $\infty^{p}:=\infty$

$$
q_{p}(k):=\left(q\left(k^{p}\right)\right)^{1 / p} \text { if } p>1, \quad:=q\left(k^{p}\right) \text { if } 0<p \leqslant 1, \quad k \in+\overline{\mathbb{R}}^{X}
$$


LEMMA 12. If $q: \overline{\mathbb{R}}^{X} \rightarrow+\overline{\mathbb{R}}$ is an integral metric with $q(2 k)=2 q(k), 0<p<$ $\infty$, then $q_{p}$ is also an integral metric on $+\overline{\mathbb{R}}^{X}$, positive-homogeneous if $p \geqslant 1$.

Proof: $2 q(k) \leqslant q(2 k)$ implies $q(t k)=t q(k), 0<t<\infty ;|s+t|^{p} \leqslant s^{p}+t^{p}$ if $p \leqslant 1$. If $p>1, q_{p}$ satisfies Minkowski's inequality for finitely-valued $k, l$ by Bourbaki [5, p.12]; if $k, l \in+\overline{\mathbb{R}}^{X}$ with finite $q_{p},\left(q_{p}(k+l)\right)^{p} \leqslant q\left(2^{p}\left(k^{p}+l^{p}\right)\right)<\infty$ and $m q_{p}\left(k_{\infty}\right)=q_{p}\left(m k_{\infty}\right) \leqslant q_{p}(k)<\infty, q_{p}\left(k_{\infty}\right)=0$ (see Corollary II); therefore $q_{p}(k+l) \leqslant q_{p}\left((k+l)_{e}\right)+0 \leqslant q_{p}\left(k_{e}+l_{e}\right) \leqslant q_{p}\left(k_{e}\right)+q_{p}\left(l_{e}\right) \leqslant q_{p}(k)+q_{p}(l)$.

So for integral norms := positive-homogeneous integral metrics $q$, Section 1-2 (and Hölder) hold for $B^{q_{p}}$ and $B^{q_{p}, B}$, especially for

$$
R_{p}(I \mid B):=B^{r}, \quad r=\left(\left(I^{B}\right)_{B}\right)_{p}, \quad L_{p}(I \mid B):=B^{*}, \quad s:=\left(I_{B}\right)_{p}, \quad 0<p<\infty .
$$

If $q$ is a $B$-additive integral norm, $0<p<\infty,(+B)^{p}=+B$, then $q_{p}$ is $B$ semiadditive (for example $B=B_{\Omega}$ or $C_{0}(X, \mathbb{R})$ ); if additionally $q(h-h \wedge n) \rightarrow 0$ and $q(h \wedge 1 / h) \rightarrow 0, n \rightarrow \infty, h \in+B$, then $M_{\cap}\left(q_{q}, B\right)=M_{\cap}(q, B), k_{j} \rightarrow k\left(q_{p}, B\right) \Leftrightarrow$ $k_{j} \rightarrow k(q, B)$,

$$
B^{q_{p}}=\left\{f \in M_{\cap}(q, B): q_{p}(|f|)<\infty\right\} .
$$

EXAmple: \|\|$_{p}=\left(\|\|_{1}\right)_{p}$ is a $+\overline{\mathbb{R}}^{X}$-semiadditive integral metric, $0<p<\infty$, $\|k\|_{1}=\sum_{x \in X} k(x)$. One can also show that $q \circ \beta_{p}$ is an integral metric on $+\overline{\mathbb{R}}^{X}$ for $0<p<\infty$ if $q$ is, $\beta_{p}(k):=\left(k^{p} /\left(1+k^{p}\right)\right)^{1 / p}$, though $\beta_{1}$ is not homogeneous.

G, SchÄFKe COMPLetions. If one has $q B 0$ and $q \sigma$-subadditive $\left(k, k_{n} \in+\overline{\mathbb{R}}^{X}\right.$, $k \leqslant \sum_{1}^{\infty} k_{n} \Rightarrow q(k) \leqslant \sum_{1}^{\infty} q\left(k_{n}\right)$ starke Integralnorm in [16]), then $B^{q}$ of Theorem 1 has by Schäfke $[16,19]$ additional properties: For $P \subset X, q(1 P)=0 \Leftrightarrow q(\infty P)=0 \Leftrightarrow$ there is $k \in N_{q}$ with $P \subset\{x \in X: k(x) \neq 0\} \Leftrightarrow P$-nullsets; $P_{n} q$-nullsets $\Rightarrow \bigcup_{1}^{\infty} P_{n}$ $q$-nullset; $\left(B^{q}, q\right)$ is complete (Stone's axiom is not needed); $q_{B}$ is also $\sigma$-subadditive, with Corollary II one gets

$$
B^{q_{B}}=B^{q}+N_{q_{B}}:=\left\{g+l: g \in B^{q}, l \in N_{q_{B}}\right\} .
$$

$q$ is called weakly $B$-semiadditive [16] if $h_{n}, h \in+B$ with $\sum_{1}^{\infty} h_{n} \leqslant h$ and $q(h)<\infty$ implies $q\left(h_{n}\right) \rightarrow 0$.

LEMma 13. If $q B 0$ holds, $q \neq \infty$ on $+B, q$ is $\sigma$-subaditive and weakly $B$ semiadditive, $f_{n} \in M_{n}(q, B), k \in \overline{\mathbb{R}}^{X}$ and $f_{n} \rightarrow k$ q $q_{B}$-almost everywhere, then 
$k \in M_{\cap}(q, B)$ if additionally the $f_{B} \in B^{q_{B}}$ and $k-f_{n} \rightarrow 0 q_{B}$-almost everywhere, then $f_{n} \rightarrow k(q, B)$.

Proof: With Lemma 4, Corollary II and the above one can assume $0 \leqslant f_{n}$ and $f_{n} \rightarrow k$, respectively $k-f_{n} \rightarrow 0$ on $X$. If $h \in+B, l_{n}:=f_{n} \wedge h$, then $p_{n}:=h-\vee_{n}^{\infty} l_{j} \in$ $B^{q}$ and $h-k \wedge h=\vee_{1}^{\infty} p+n \in B^{q}$ by Hilfssatz 1.4.4 of Schäfle [16], so $k \in M_{\cap}(q, B)$. For the second assertion, $\beta_{n}:=\left|k-f_{n}\right| \wedge h \in B^{q}$ by Lemma 4 and Theorem $2, \beta_{n} \rightarrow 0$; [16], Satz 1.4.6 gives $q\left(\beta_{n}\right) \rightarrow 0$. (See also Liubicich [12]).

If only $f_{n} \rightarrow k \quad q_{B}$-almost everywhere, but $\left|f_{n}\right| \leqslant g_{0} \in B^{q_{B}}$ for $n \in \mathbb{N}$, or if $g_{0} \leqslant f_{n} \leqslant f_{n+1}, \sup q_{B}\left(f_{n}-g_{0}\right)<\infty$ and $q$ is $B$-semiadditive, one gets immediately $k-f_{n} \rightarrow 0 \quad q_{B}$-almost everywhere, then the usual $L^{1}$-convergence theorems follow from those of Sections 1 and 2. (For nets they and Lemma 17 are false.)

H, Daniell integrals. If $I \mid B$ with (9) is $\sigma$-continuous, that is $I\left(h_{n}\right) \rightarrow 0$ whenever $h_{n} \in+B \quad h_{n} \geqslant h_{n+1} \rightarrow 0$ pointwise on $X$. Then Aumann $[1$, p.448-449]

$$
I^{\sigma}(k):=\inf \left\{\sum_{1}^{\infty} I\left(h_{n}\right): k \leqslant \sum_{1}^{\infty} h_{n}, h_{n} \in+B\right\}, \quad k \in+\overline{\mathbb{R}}^{X}
$$

defined a $\sigma$-subadditive $B$-additive integral norm with $q\left(k_{n}\right) \rightarrow q(k)$ for any $k_{n}, k \in$ $+\overline{\mathbb{R}}^{X}$ with $k_{n} \uparrow k, q=I^{\sigma}$ or $I_{B}^{\sigma}$, (= upper $S$-norm of Bichteler [2]); so all results of Sections 1-3 and $G$ hold for $\left.I_{B}^{\sigma}:=\left(I^{\sigma}\right)_{B}\right)$

$$
L^{1}:=B^{1}, \quad L_{1}:=B^{I_{B}^{\sigma}}, \quad I:=I_{D}:=I^{I_{B}^{\sigma}}, \quad\|f\|_{1}:=I_{D}(\|f\|)
$$

$I_{D} \mid L^{1}=$ usual Daniell extension of $I \mid B,[6,1,15,9]$.

As an analogue to $\bar{B}=L^{1} \cap \bar{B}+\bar{B}_{N}$ of [11] we can only show

$$
\begin{aligned}
& \mu \sigma \text {-additive on } \delta \text {-ring } \Omega \Rightarrow L_{1}=R_{1} \Rightarrow L^{1}=\bar{B} \cap R_{1}+L_{1, N} \\
& \Rightarrow L^{1} \subset \bar{B}+L_{1, N} \Rightarrow L=\bar{B}+L_{N} \Rightarrow L=L^{1} \cap L+L_{N}
\end{aligned}
$$

with $M_{N}:=\{$ null functions of $M$ \}. So at least for measure spaces $(X, \Omega, \mu)$ and $I\left|B=I_{\mu}\right| B_{\mu}$ one has

$$
L^{1} \subset L=\bar{B} \cap L^{1}+L_{N}=L \cap L^{1}+L_{N}
$$

even then $L_{1} \varsubsetneqq \bar{B}$ is possible ([11]).

As an analogue to the first $\Rightarrow$ of $(40)$ one has $L_{1}=R_{1}$ for $\sigma$-continuous $I \mid B$ satisfying $\sum_{1}^{\infty} h_{n} \in B$ whenever $h_{n} \in+B$ with $\sum_{1}^{\infty} h_{n} \leqslant h \in B$. 
I, BouRbaKI INTEgRaLs. If $I \mid B$ with (9) is $\tau$-continuous $\left(I\left(h_{j}\right) \rightarrow 0\right.$ if $\left(h_{j}\right)$ net from $B$ with $\left.h_{j} \downarrow 0\right)$ then $B^{+}=B_{+}, I^{+}=\bar{I}$ on $B^{+}, I^{r}:=\left(I^{+}\right)^{B^{+}}=\bar{I} \leqslant I^{\sigma}$ defines a $B$-additive integral norm on $+\overline{\mathbb{R}}^{X}$ with $I^{\tau} B I[3,11]$, so $L^{\tau}:=B^{\Gamma^{\tau}}$ and $L_{\tau}:=B^{I_{B}^{\tau}}$ are with their integrals well defined, $L^{r}=$ Bourbaki-integrable functions (Pfeffer [15], Floret $\left[9\right.$, p.338]; see also \|\|$_{4}$ of Schäfke [16]) one has

$$
L^{1} \subset L^{\tau}=\bar{B}=L^{1}+\bar{B}_{N} \subset L_{\tau}=L=L^{1}+L_{N}=L_{1}+L_{N} .
$$

Examples: $B=C_{0}(X, \mathbb{R}), X$ Hausdorff, or $I_{\mu} \mid B_{\mathbf{n}}, \mu=$ Lebesgue measure, $\Omega=$ intervals $\subset X$ open $\subset \mathbb{R}^{n}$ (here $L^{1}=L$ by [11]); even then the integral is not $\tau$-continuous on $L^{1}$. There are $I_{\mu} \mid B_{\Omega}$ with $1 X \in B_{\Omega}^{+}$and all " $C$ " in (44) are strict.

\section{REFERENCES}

[1] G. Aumann, 'Integralerweiterungen mittels Normen', Arch. Math. 3 (1952), 441-450.

[2] K. Bichteler, Integration theory, Lecture Notes in Mathematics 315 (Springer-Verlag, Berlin, Heidelberg, New York, 1973).

[3] P. Bobillo Guerrero and M. Diaz Carrillo, 'Summable and integrable functions with respect to any Loomis system', Arch. Math. 49 (1987), 245-356.

[4] P. Bobillo Guerrero and M. Diaz Carrillo, 'On the summability of certain $\mu$-integrable functions', Arch. Math. 52 (1989), 258-264.

[5] N. Bourbaki, Intégration. Elements de Mathematique XIII, Livre VI, (Hermann, Paris, 1952).

[6] P.J. Daniell, 'A general form of Integral', Ann. of Math. 19 (1917), 279-294.

[7] M. Díaz Carrillo and H. Günzler, 'Finitely additive integration II', Extracta Mathematicae 4 (1989), 81-83.

[8] N. Dunford and J.T. Schwartz, Linear operators I (Interscience, New York, 1957).

[9] K. Floret, Maß- und Integrationstheorie, (Teubner, Stuttgart, 1981).

[10] H. Günzler, Integration (Bibliogr. Institut, Mannheim, 1985).

[11] H. Günzler, 'Convergence theorems for a Daniell-Loomis integral', Mathematica Pannonica 2 (1991), 77-94.

[12] P. Liubicich, 'Sul prolungamento dell'integrale', Rend. Instit Mat. Univ. Triestre 8 (1976), 108-121.

[13] L.H. Loomis, 'Linear functionals and content', Amer. J. Math. 76 (1954), 168-182.

[14] P. Muñoz Rivas, 'Integración finitamente aditiva: extensión integral con convergencia I-local', Serv. Publ. Univ. Granada Gr-1695 (1990).

[15] W.F. Pfeffer, Integrals and measures, (Dekker, New York, 1977).

[16] F.W. Schäfke, 'Integrationstheorie I.', J. Reine Angew. Math. 244 (1970), 154-176.

[17] F.W. Schäfke, 'Integrationstheorie II', J. Reine Angew. Math. 248 (1971), 147-171.

[18] F.W. Schäfke, 'Integrationstheorie und quasinormierte Gruppen', J. Reine Angew. Math. 253 (1972), 117-137. 
[19] F.W. Schäfke, 'Lokale Integralnormen und verallgemeinerte uneigentliche Riemann- Stieltjes-Integrale', J. Reine Angew. Math. 289 (1977), 118-134.

\author{
Dpto Analisis Matematico \\ Universidad de Granada \\ Granada \\ Spain
}

\author{
Mathematisches Seminar \\ Der Christian-Albrechts-Universität \\ Kiel \\ Germany
}

